\title{
Surgical.
}

\section{THE TREATMENT OF THORACIC EMPYEMA.}

\author{
BY R. SLEIGH JOHNSON, M.D., M.R.C.P.LOND. \\ Physician-in-Charge of Out-Patients, City of London Hospital for Diseases of the Heart and Lungs; \\ Casualty Physician to St. Bartholomere's Hospital.
}

FEw medical conditions have in the past aroused more controversy than the treatment of thoracic empyema. During the last hundred years the pendulum of opinion has swung from an almost complete neglect of active measures, when all but a fortunate few who recovered by spontaneous rupture were left to die, to an indiscriminating routine at the beginning of this century of immediate rib-resection and open drainage in all cases. Within the last thirty years it has gradually been realized that thoracic empyema is not a surgical emergency, and that no one method of treatment is applicable to every case. An attempt is made in this article to consider the varieties of empyema commonly met, and, while omitting details of surgical technique, to outline in each instance the appropriate mode of treatment.

A practical classification of infections of the pleura in the adult may be made under the headings-

(I) Pyogenic infections: (a) acute fulminating empyema; (b) post-pneumonic empyema.

(2) Tuberculous infections: (a) "sterile"; (b) mixed infection.

These cover the great majority of cases; in rare instances other infections are found, e.g., by the streptothrix group of organisms. Special considerations applying to the treatment of young children will be discussed later.

1. Pyogenic

(a) Acute fulminating empyema. If injury as a cause be excluded,

Infections. suppuration due to pyogenic organisms in the pleural cavity is almost invariably associated with a pneumonic process in the underlying lung. When the infecting organism is of high virulence, frequently a streptococcus alone or in symbiosis, the spread of infection through the lung substance is very rapid and the barrier of resistance offered by the visceral pleura is readily overcome. Simultaneously with consolidation of the lung there occurs a rapid outpouring of a thin turbid exudate into the pleura, forming the so-called "syn-pneumonic empyema." The picture is seen most frequently in influenzal infections and in pneumonia of the "septic" variety such as follows aspiration of infected material or a foreign body into a bronchus.

It is in this type of empyema that any treatment by rib resection and open drainage is disastrous. The appalling risk of such measures was emphasized during the war by the experience of influenza epidemics in some American camps. Acute fulminating empyema was a frequent complication in such cases, and the mortality following immediate operation varied between 40 and 60 per cent. 
The reasons for this grave risk of open operation are not far to seek. The empyema in these cases develops early in the course of the disease, sometimes before definite $\underset{\infty}{z}$ consolidation of the lung has occurred. The inflammatory changes in the lung are intense, with much congestion and cedema. The general state of the patient is one of grave illness, and respiratory embarrassment is great. The effusion into the pleura is at first a thin turbid fluid, sometimes offensive, of a dirty brown or greenish colour, with no evidence of fibrin production. The affected pleural cavity becomes converted into a pyothorax, since no adhesions are formed at this stage and no protective fixation of the mediastinal structures occurs. If a large opening is made into the pleural cavity on the side of the empyema, with each inspiratory effort the mediastinum is drawn over to the $\vec{o}$ opposite sound side, restricting the expansion of that lung, only to be forced back again past its original position by the succeeding expiration.

This to-and-fro pendulum-like movement of the mediastinal contents, or "mediastinal flutter," still further hampers the heart and great vessels and reduces the already impaired oxygenating power of the lungs. The gravity of the patient's illness is necessarily greatly increased and death from heart failure with increasing cyanosis and dyspnœa commonly ensues.

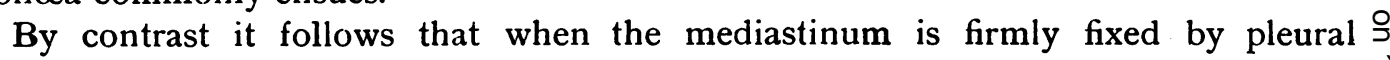
adhesions, i.e., when a localized collection of frank pus has formed, these dangers are minimized. Such is the case in the common variety of post-pneumonic empyema of pneumococcal origin, which is rarely recognized until after the crisis, by which time adhesions have formed and operation is comparatively safe.

In the treatment of acute streptococcal empyema, therefore, an initial aspiration should be the first stage. The exudate is usually thin and free from obstructing masses of fibrin, and is readily removed with exploring needle and syringe. Because of it toxic character as much fluid should be taken off as can be easily obtained without causing symptoms of distress, though if the empyema be very large the removal is best done in stages. A word of caution may be given against the production of too great a negative pressure in aspiration, shown by an increase of cyanosis and dyspnœea and complaint of pain in the chest. If such symptoms arise they should be relieved by the cautious adrnission of a small amount of air through the disconnected needle, the air being filtered through a small plug of cotton-wool placed over its opening. Aspiration should be repeated daily or every other day according as the toxic symptoms are relieved, until the pus becomes too thick to run through the needle. Not until frank pus has appeared, i.e., perhaps for two or three weeks, can rib resection safely be performed.

In some cases where the condition of the patient will allow, a primary aspiration of the pus may be followed by the institution of "closed" or negative-pressure drainage. The aim of such treatment is to drain the empyema cavity by continuous suction, and to keep it empty until the underlying lung has fully re-expanded and discharge has ceased. The maintenance of a negative pressure in the affected pleura effectively prevents displacement of the mediastinum. A number of methods are available for the purpose, and local anæsthesia is all that is required. The simplest contrivance is the introduction with a trocar of a rubber catheter leading by a length of rubber 0 tubing below the surface of a bowl of weak antiseptic fluid under the patient's bed. The catheter may be maintained in position by successive rings of collodion gauze, or 
may be held in place by introduction through a cannula attached by tapes to the chest wall. In either case the connection to the chest must be air-tight.

Other devices for maintaining a negative pressure can be made by connecting the catheter to a suction pump, intercepted by a receiving bottle in which the pus is retained, or to a simple arrangement of aspirating bottles. The suction exerted by the syphon action keeps the discharge constantly flowing into the receiving bottle. These methods require supervision to guard against occasional blocking of the tubing, an event which may usually be rectified by careful clearing with a syringe. In cases where the pus is thick or large masses of fibrin are present they are definitely unsuitable. Daily irrigation, or in some cases even continuous irrigation of the cavity with Dakin's solution (0.5 per cent. sodium hypochlorite) should be carried out; this has the merit of softening any masses of fibrin present and of minimizing the thickening of the pleura which otherwise results from fibrin deposition in layers on its surface. Drainage should be maintained until the discharge becomes slight and serous, fever is absent, and the expansion of the lung approaches normal. Great care must be taken throughout to avoid secondary infection of the wound with resultant delay in healing. Besides promoting early expansion of the lung negative-pressure drainage has the advantage of greater comfort to the patient. Frequent changes of dressing are avoided, and the pain and discomfort of coughing induced by the sucking in of air through exposure of an open tube are abolished.

(b) Post-pneumonic Empyema.-Pneumococcal empyema seldom becomes apparent until after the crisis. Usually by the time the diagnosis is made adhesions have already formed, so that thoracotomy and rib resection may be safely carried out. In contrast with streptococcal effusions the pus is usually thick and creamy in consistence with large masses of fibrin. For this reason treatment by any closed method of drainage is likely to fail. For the ordinary case rib resection and the insertion of a very large bore tube, carefully sewn in, gives excellent results. The tube should extend only just into the pleural cavity and must not press on the diaphragm; the site of incision must, however, be low enough to permit of and maintain adequate emptying of the cavity. The eighth or ninth rib in the posterior axillary line is frequently a convenient site to choose. Local anæsthesia is preferable, and if necessary may be supplemented by gas and oxygen. Daily irrigation with Dakin's solution should be carried out at least in the early stages of drainage.

Less commonly, however, the presence of a pneumococcal empyema is recognized early in the illness, while the persistence of pneumonia is shown by a raised respiratory rate with cyanosis and dyspnœa. In these cases a primary aspiration is advisable, followed by an attempt to establish closed negative pressure drainage. Occasionally where the effusion is small or consists merely of turbid serum, recovery may be complete after aspiration alone, but this is not to be relied on and the procedure should be regarded as a temporary expedient only. Aspiration should also be adopted as a preliminary measure : $(a)$ when the empyema is very large; $(b)$ in grave illness; $(c)$ in infancy and old age.

Other methods of treatment of pneumococcal empyema have been advocated. Simple intercostal incision and immediate closure of the wound without drainage generally fails in its object and adequate drainage is needed later. Similarly, intercostal incision and insertion of a rubber drainage tube without rib resection, while 
affording temporary relief, seldom gives a free enough exit to the pus to allow of healing.

Special considerations apply to the treatment of empyema in young children. The duration of pneumonia in the infant is apt to be more prolonged than in the adult, so that the co-existence of effusion and consolidated lung is of more frequent occurrence. Moreover, in young children it is well known that the two conditions may closely resemble each other or even be indistinguishable in their physical signs. Whenever, therefore, the presence of fluid is suspected, an exploratory puncture of the chest should be made, for there is no other certain means of confirming its presence or diagnosing its nature. Especially is this so in the case of young children in whom the majority of pleural effusions are purulent in character. Delay in diagnosis and treatment of an empyema is the most common cause of its failure to close. Moreover, an opportunity is afforded by puncture of determining the nature of the infecting organism.

Young children withstand badly any considerable displacement of the heart or sudden alteration of pleural pressure, and the mortality of rib resection and open drainage below the age of 2 years is so high as to exclude it as a method of treatment. The pus should be aspirated under local anæsthesia, followed by continuous suction drainage to evacuate the empyema gradually and to keep the cavity empty until the lung has fully expanded.

In post-pneumonic cases over the age of 2 years there is less objection to rib resection, but a preliminary aspiration twelve hours or so earlier should be made te minimize displacement of the mediastinum and respiratory embarrassment. A localo izing test on the operating table with an exploring needle should always precede incisions

Empyema in children is occasionally bilateral. In this case aspiration should bêे the treatment adopted, of the more acutely affected side first, then of the other. When definite pus has formed negative pressure drainage should be established on both sides.

The most important points in after-treatment, both in the child and in the adult, are fresh air, good food, the maintenance of adequate drainage and the promotion of early expansion of the lung. This may be aided by breathing exercises while compressing the sound side of the chest, blowing exercises with Woulfe's bottles, and by allowing the patient up as early as his condition will permit. Country or seaside convalescence aids in restoring the full degree of chest expansion.

In uncomplicated cases healing should be complete in from four to six weeks. An empyema may be deemed chronic when it has failed to close within three months of operation. By far the most common cause is inadequacy of drainage, and the first step is therefore to enlarge the opening by resection of portions of one or two adjacent ribs. Any loculi of pus must be opened up; a preliminary injection into the sinus of a substance opaque to X-rays, such as lipiodol or barium emulsion, may assist in localizing any such tracks present. Occasionally a foreign body is the cause of the delay in healing and if found should be removed. If the cavity still fails to close in spite of all efforts to get the lung to expand, it may be necessary to bring the chest wall to the lung by means of a thoracoplasty. Decortication or stripping of the thickened sheet of visceral pleura from the lung is sometimes successful but is a formidable operation and not devoid of risk of tearing the lung substance. A plastic operation of some degree is generally to be preferred. 
Brief mention must be made of interlobar empyema. This is difficult to distinguish from abscess of the lung, and indeed frequently remains undiagnosed until rupture occurs into a bronchus. Physical signs may be slight or absent and the condition suspected solely from the gravity of the symptoms. If the abscess grows to any size without rupture an area of dullness and weakened breath sound may sometimes be detected in the axilla. The X-ray appearance may assist diagnosis, showing a dense shadow along the line of the interlobar septum.

Treatment is as for a lung abscess, by exposure and external drainage. Unless the pus is obviously near the surface exploration with a needle is dangerous, involving a considerable risk of causing a generalized empyema. Great care must be taken at operation that the general pleural cavity is shut off by adhesions before the pus is located and drained; otherwise the lung must be first stitched to the parietal pleura and the actual opening preferably delayed for a few days. If rupture has already occurred into the lung or a bronchus, aspiration through a bronchoscope may assist recovery.

\section{Tuberculous}

Empyema.
Tuberculous empyemata fall into two groups :-

(a) Those in which secondary infection is absent, though tubercle bacilli are usually present in large numbers. This type may occur of itself or may complicate artificial pneumothorax treatment, although in the great majority of the latter cases effusion is serous in character. Open drainage is the worst possibie treatment; healing does not occur and secondary infection at some stage is almost inevitable. The pus should be aspirated completely and if of large amount replaced by air. The risk of a needle-track with subsequent sinus formation can be minimized by disinfecting the exploring needle with iodine. Care should also be taken to avoid making a puncture too far posteriorly in view of the possible necessity later of thoracoplasty. If the pus is thick and difficult to remove it may be rendered more fluid by the injection on the previous day of Io to 20 c.c. of Gauvain's modifying fluid.

Should the pus re-accumulate after repeated air replacement, the pleural cavity may be gently washed out with normal saline or a weak antiseptic solution, such as methyleneblue $I$ in 4,00o or dilute acriflavine or iodine. If in spite of these measures the empyema persists and the condition of the other lung permits, thoracoplasty preceded by phrenic evulsion will have to be considered as a possible means of obliterating the cavity.

(b) A secondarily infected tuberculous empyema is a grave event and usually secondary to a spontaneous pneumothorax. Treatment is difficult and frequently of little avail. An attempt should be made to clean up the cavity as far as possible and to avoid open drainage. Irrigation of the pleural cavity through two needles may occasionally clear up the secondary infection if this be by organisms of only feeble virulence. In most cases closed negative-pressure drainage will be required, and in some rib resection cannot be avoided. In these, however, it is with the knowledge that healing never occurs and a stage of gradual deterioration leads inexorably to a fatal issue. 